\title{
Small-angle neutron-scattering studies on nonaqueous dispersions Part 5: Magnesium carbonate dispersions in hydrocarbon media
}

\author{
R. H. Ottewill, E. Sinagra, I. P. MacDonald ${ }^{1}$ ), J. F. Marsh ${ }^{1}$ ), and R. K. Heenan ${ }^{2}$ ) \\ School of Chemistry, University of Bristol, Bristol, UK \\ 1) Exxon Chemical Limited, Abingdon, Oxfordshire, UK \\ 2) ISIS, Rutherford-Appleton Laboratories, Chilton, Oxfordshire, UK
}

\begin{abstract}
A method is described for the preparation of magnesium carbonate particles in a nonaqueous hydrocarbon environment. The particles were stabilised in the colloidal sense by the adsorption of an alkyl-aryl sulphonic acid. The particle morphology and particle-size distribution were examined by small-angle neutron scattering. It was concluded that the particle structure was of the coreshell type, with a core particle of basic magnesium carbonate, radius ca. $40 \AA$, and a shell of thickness ca. $13 \AA$. The results suggested that the latter was predominantly that of the alkyl-aryl part of the stabilising moiety.
\end{abstract}

Key words: Magnesium carbonate dispersions; core-shell morphology; smallangle neutron scattering; nonaqueous dispersions

\section{Introduction}

Dispersions of inorganic materials in nonaqueous media are widely used in industrial practice and, in this context, alkaline earth carbonates stabilised by surface-active agents form an important group. They are frequently used as additives to lubricating oils in order to neutralise acids formed during the combustion of sulphur-containing fuel in internal combustion engines. The technical aspects of this process have been considered elsewhere [1-4].

From the viewpoint of the colloid scientist, they form an interesting group of model dispersions that contain particles of the core-shell type, i.e., a core particle of the alkaline earth carbonate surrounded by a shell of a surface-active agent. There is, therefore, considerable interest in the structure of the particles, the origin of their colloid stability [5] and their behaviour in concentrated dispersions [6].

In previous work, parts 1 to 4 [7-10], we have investigated the nature and behaviour of calcium carbonate particles stabilised by surface-active agents in both aromatic and aliphatic hydrocarbons. It was found in this work that the size-range of the particles, and the magnitudes of the coherent scattering length of the core and the shell made them excellent systems for study by small-angle neutron scattering. An additional useful feature was the ability to examine the systems in mixtures of hydrogenated and deuterated dispersion media $[7,8,11,12]$.

In this work we have examined the preparation and properties of magnesium carbonate particles stabilised by an alkyl-aryl sulphonic acid in an aliphatic hydrocarbon.

\section{Experimental}

\section{Materials}

$h_{18}$-octane was $\mathrm{BDH}$ laboratory grade material.

$d_{18}$-octane was obtained from the Aldrich Chemical Company as $98+$ atom \% D

The methanol, acetone, heptane and toluene were all hydrogenated-materials and were obtained as $\mathrm{BDH}$ laboratory grade solvents.

The surface-active agent was an alkyl-aryl sulphonic acid of molecular weight ca. 500. This material and a commercialgrade hydrocarbon with a viscosity of 30 centistokes at $40^{\circ} \mathrm{C}$ were supplied by Exxon Chemical. 


\section{Apparatus}

This consisted of a round-bottomed flask equipped with five inlets. Through the various inlets were inserted a copperconstantan thermocouple, a stirring paddle, a gas-distribution tube and a condenser. The thermocouple was connected to a West Gardian temperature-control unit which monitored and maintained the pre-selected reaction temperature. This was in turn connected to the heating mantle which contained the flask.

\section{Preparation of magnesium carbonate dispersions}

A mixture of an alkyl-aryl sulphonic acid $(365 \mathrm{~g})$ and toluene $(720 \mathrm{~g})$ was placed in the reaction vessel and homogenised by stirring. Methanol ( $13 \mathrm{~g}$ ) was added and the temperature was stabilised at $25^{\circ} \mathrm{C}$. Magnesium oxide ( $154 \mathrm{~g}$ ) was added to this mixture over a period of $10 \mathrm{~min}$, during which time the temperature rose and was stabilised at $40^{\circ} \mathrm{C}$ once all the oxide had been added. A solution of an amine catalyst $(89 \mathrm{~g})$ was then added, followed by a mixture of methanol ( $82.5 \mathrm{~g}$ ) and water $(110 \mathrm{~g})$. Carbon dioxide ( $175 \mathrm{~g})$ was then passed through the aerator in the mixture at a rate of $39 \mathrm{~g} / \mathrm{h}$. This was an exothermic process and the temperature increased to above $60^{\circ} \mathrm{C}$. On completion of the carbonation, a commercial-grade hydrocarbon solvent was added. The temperature was then raised in a regulated manner and a condenser was fitted to the reaction vessel for a distillation. The distillation process was then continued under vacuum at a temperature of $165^{\circ} \mathrm{C}$ for 105 min until the methanol, water and toluene had been driven off. Finally, a small amount of filter-aid was added to the hot mixture and it was filtered under pressure.

In order to concentrate the sample, the colloidal particles were diluted by heptane and acetone added until the mixture became turbid. The precipitate formed was centrifuged and the supernatant discarded. The sediment was then rotary evaporated to remove the heptane and acetone. The remaining residue was a glassy form and by titration against $0.1 \mathrm{~mol} \mathrm{dm}^{-3}$ perchloric acid the concentration of magnesium carbonate was found to be $47.5 \% \mathrm{wt} / \mathrm{wt}$. The samples for scattering studies were prepared by redispersing known amounts of the solid material in appropriate mixtures of $b_{18}$ octane and $d_{18 \text {-octane. }}$

\section{Small-angle neutron scattering}

All the scattering measurements were carried out using the LOQ diffractometer at the ISIS unit of the RutherfordAppleton Laboratory [13]. The samples were examined in optical-quality quartz cells having a path length of $1 \mathrm{~mm}$. LOQ is a time-of-flight instrument operated from a pulsed source of neutrons. An area detector $4.2 \mathrm{~m}$ from the sample collected data for neutron wavelengths of $2.0-10.0 \AA$. After allowing for the wavelength dependence of the sample transmission and the detector efficiencies, absolute scattering intensities $\partial \Sigma / \partial \Omega$ were obtained [13] as a function of $Q$. The scattering vector of magnitude $Q$ was defined by

$$
Q=4 \pi \sin (\theta / 2) / \lambda,
$$

with $\theta$ being the scattering angle. The $Q$-range examined was ca. 0.01 to $0.23 \AA^{-1}$.

\section{Concentric sphere particles}

For this geometry the core particle can be defined (as previously $[7,8])$ as having a radius $R_{\mathrm{c}}$ and a coherent neutron-scattering length density of $\rho_{c}$, with an outer shell of thickness $\delta$, and a coherent scattering length density $\rho_{\mathrm{s}}$. Thus, the total radius of the particle $R_{\mathrm{T}}$ is given by $R_{\mathrm{c}}+\delta$.

The basic scattering equation for the intensity of scattering of a system of $N_{p}$ concentric-sphere particles can be written in the form

$$
\left[\frac{d \Sigma}{d \Omega}(Q)\right]=N_{\mathrm{p}}\left[\left(\rho_{\mathrm{s}}-\rho_{\mathrm{m}}\right)\left(A_{2}-A_{1}\right)+\left(\rho_{\mathrm{c}}-\rho_{\mathrm{m}}\right) A_{1}\right]^{2},
$$

where $\rho_{\mathrm{m}}$ is the coherent scattering length density of the medium. $A_{1}$ and $A_{2}$ are defined by

$$
A_{1}=3 V_{\mathrm{c}}\left[\left(\sin Q R_{\mathrm{c}}-Q R_{\mathrm{c}} \cos Q R_{\mathrm{c}}\right) / Q^{3} R_{\mathrm{c}}^{3}\right] \text {, }
$$

and

$$
A_{2}=3 V_{\mathrm{T}}\left[\left(\sin Q R_{\mathrm{T}}-Q R_{\mathrm{T}} \cos Q R_{\mathrm{T}}\right) / Q^{3} R_{T}^{3}\right],
$$

such that,

$$
V_{\mathrm{T}}=4 \pi\left(R_{\mathrm{c}}+\delta\right)^{3 / 3} \text { and } V_{\mathrm{c}}=4 \pi R_{\mathrm{c}}^{3} / 3
$$

The volume fraction of the system can also be defined as

$$
\phi=N_{\mathrm{p}} V_{\mathrm{T}} /\left(N_{\mathrm{p}} V_{\mathrm{T}}+V_{\mathrm{m}}\right) \approx N_{\mathrm{P}} V_{\mathrm{T}} / V_{\mathrm{m}}
$$

for dilute dispersions with $V_{\mathrm{m}}$ being the volume of the dispersion medium.

\section{Polydispersity}

Polydispersity of the core particles was taken into account using a zeroth-order log normal distribution, namely,

$$
p\left(R_{\mathrm{c}}\right)=\frac{\exp \left[-\left(\ln R_{\mathrm{c}}-\ln R_{\mathrm{m}}\right)^{2} / 2 \sigma_{0}^{2}\right]}{(2 \pi)^{1 / 2} \sigma_{0} R_{\mathrm{m}} \exp \left(\sigma_{0}^{2} / 2\right)},
$$

where $p\left\langle R_{\mathrm{c}}\right\rangle$ gives the proportion of core particles of radius $R_{\mathrm{c}}$ with $R_{\mathrm{m}}$ the mean modal core radius and $\sigma_{0}$ a parameter describing the width and skewness of the distribution [14].

\section{Results}

\section{Materials}

The basic materials used in this work were particles of magnesium carbonate, an alkyl-aryl sulphonic acid, $h_{18}$-octane and $d_{18}$-octane. The chemical formulae and coherent neutron scattering densities $\rho$ for these materials are listed in Table 1 .

\section{SANS studies}

The results obtained on samples containing $1 \%$ of magnesium carbonate by weight in $h_{18}$-octane, $60 \% d$-octane $+40 \% h_{18}$-octane, $80 \% d_{18}$-octane 
Table 1. Coherent neutron scattering densities, $\rho$

\begin{tabular}{llll}
\hline Material & Formula & density $/ \mathrm{g} \mathrm{cm}^{-3} \rho / 10^{10} \mathrm{~cm}^{-2}$ \\
\hline Magnesium & $3 \mathrm{MgCO}_{3} \mathrm{Mg}(\mathrm{OH})_{2}$ & \\
Carbonate & $3 \mathrm{H}_{2} \mathrm{O}$ & 2.16 & 3.34 \\
Surfactant & $\mathrm{C}_{30} \mathrm{H}_{53} \mathrm{SO}_{3}^{-\ddagger}$ & $1.00^{\dagger}$ & 0.25 \\
$h_{18}$-octane & $\mathrm{C}_{8} \mathrm{H}_{18}$ & 0.7036 & -0.524 \\
$d_{18}$-octane & $\mathrm{C}_{8} \mathrm{D}_{18}$ & 0.815 & 6.44
\end{tabular}

${ }^{\dagger}$ approximate value

‡approximate formula

$+20 \% h_{18}$-octane and $d_{18}$-octane are plotted in Fig. 1 in the form of $[d \Sigma(Q) / d \Omega]$ against $Q$.

The experimental data are shown as open circles in the curves. These were fitted by a least-squares procedure using the formula given by Eq. (2) with due allowance for polydispersity of the core particles using Eq. (7). The continuous lines represent the fits obtained. Data points at $Q$ values below $0.01 \AA^{-1}$ were rather "noisy" and were not included in the fitting procedure. A value of 0.05 was taken for $\sigma_{0}$ but the curves were not very sensitive to variation of this quantity between 0.05 and 0.10 . The scattering length densities taken were those listed in Table 1. Thus, the curves were fitted on the two parameters $R_{\mathrm{c}}$ and $\delta$ and the values obtained are listed in Table 2. A value of the intensity of scattering at zero scattering vector, namely, $[d \Sigma(O) / d \Omega]$ was also obtained from the fits and is listed in Table 2 .

\section{The Guinier region}

As shown in previous work $[7,8]$, by expansion of the terms in Eq. (2) it can be shown that for small values of $Q R_{\mathrm{T}}$, we can write, following Guinier [15], that

$$
\left(\frac{d \Sigma}{d \Omega}(Q)\right)=\left(\frac{d \Sigma}{d \Omega}(\mathrm{O})\right) \exp \left(-R_{g}^{2} Q^{2} / 3\right\rangle
$$

where for a concentric sphere the radius of gyration is given by

$$
R_{g}^{2}=\frac{3}{5}\left\{\frac{\left(\rho_{S}-\rho_{m}\right) R_{\mathrm{T}}^{5}-\left(\rho_{C}-\rho_{S}\right) R_{\mathrm{c}}^{5}}{\left(\rho_{\mathrm{S}}-\rho_{m}\right) R_{\mathrm{T}}^{3}-\left(\rho_{C}-\rho_{S}\right) R_{\mathrm{c}}^{3}}\right\} .
$$
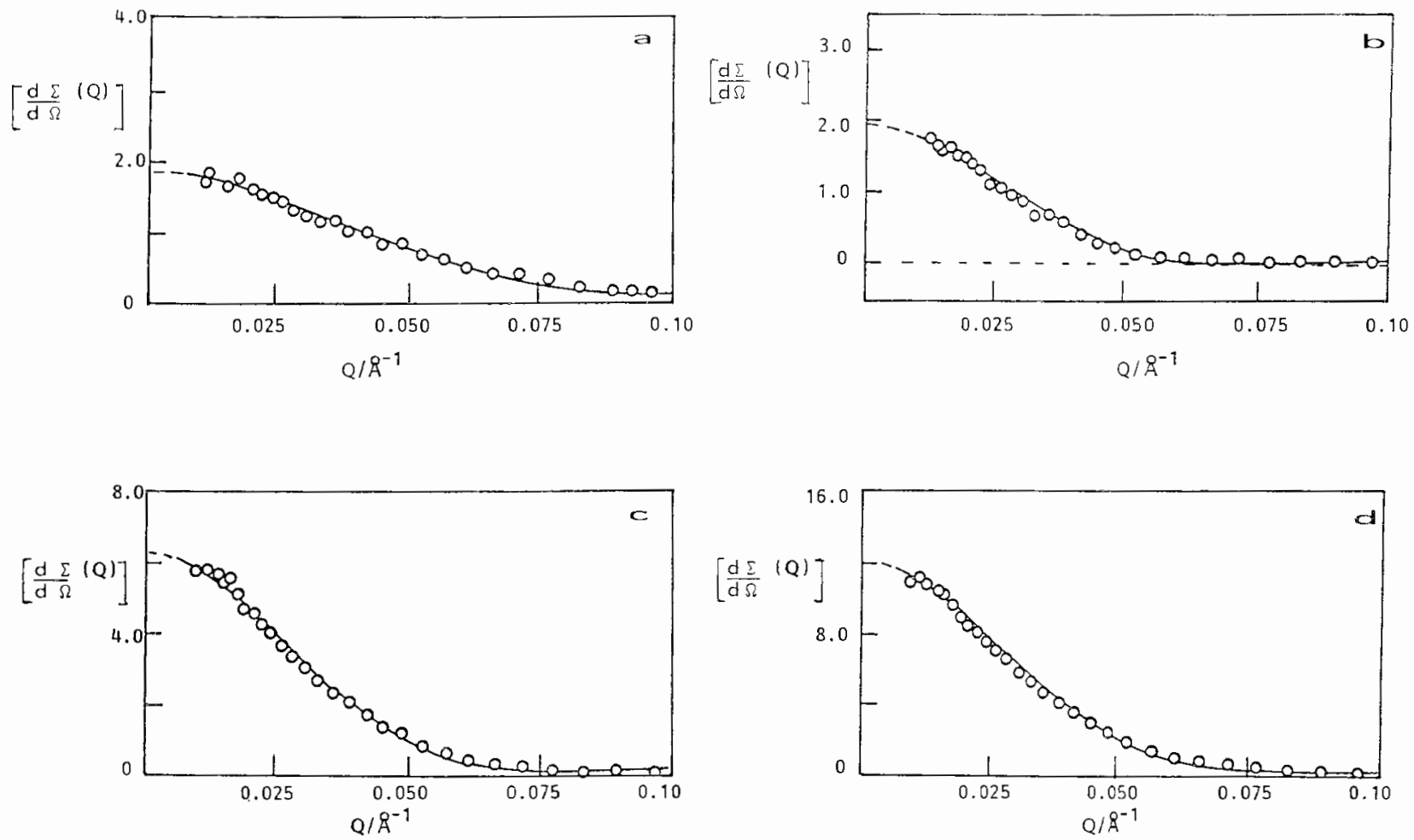

Fig. 1. Curves of $[d \Sigma(Q) / d \Omega]$ against $Q$ for $1 \% \mathrm{w} / \mathrm{w}$ magnesium carbonate dispersions in $h_{18}$-octane: $d_{18}$-octane systems a) $100 \% h_{18}$-octane, b) $60 \% d_{18}$-octane, c) $80 \% d_{18}$-octane, d) $100 \% d_{18}$-octane. $\bigcirc$, experimental results; ——, fitted curve. 
Table 2. Core-shell analysis

\begin{tabular}{|c|c|c|c|c|}
\hline Medium & $\rho_{\mathrm{m}} / 10^{10} \mathrm{~cm}^{-2}$ & $R_{\mathrm{c}} / \AA$ & $\delta / \AA$ & $\frac{d \Sigma}{d \Omega}(O)$ \\
\hline$b_{18}$-octane & -0.524 & 39.0 & 12.3 & 1.85 \\
\hline $60 \% d_{18}$-octane & 3.404 & 40.0 & 13.2 & 1.97 \\
\hline $80 \% d_{18 \text {-octane }}$ & 4.88 & 40.9 & 13.2 & 6.23 \\
\hline$d_{18}$-octane & 6.44 & 40.9 & 13.2 & 12.00 \\
\hline \multirow[t]{2}{*}{ Average } & \multirow[b]{2}{*}{ Average } & 40.2 & 13.0 & \\
\hline & & \multicolumn{2}{|c|}{$R_{\mathrm{T}}=53.2 \AA$} & \\
\hline
\end{tabular}

Table 3. Data from Guinier analysis

\begin{tabular}{lrrr}
\hline Medium & $R_{g}^{2} / \AA^{2}$ & $R_{g} / \AA$ & $\frac{d \Sigma}{d \Omega}(O)$ \\
\hline$h_{18}$-octane & 912 & 30.2 & 1.63 \\
$60 \% d_{18}$-octane & 2580 & 50.8 & 2.19 \\
$80 \% d_{18}$-octane & 2006 & 44.8 & 6.16 \\
$d_{18 \text {-octane }}$ & 1964 & 44.3 & 12.75 \\
\hline
\end{tabular}

Thus, as suggested by Guinier [15], a plot of $\ln [d \Sigma(Q) / d \Omega]$ against $Q^{2}$ allows values of $R_{g}^{2}$ and $[d \Sigma(O) / d \Omega]$ to be obtained. These values are listed in Table 3. A plot of $\ln [d \Sigma(Q) / d \Omega]$ against $Q^{2}$ is given in Fig. 2.

Since $\rho_{\mathrm{s}}$ and $\rho_{\mathrm{c}}$ are volume properties the average coherent scattering length density of a concentric sphere particle can be written as

$$
\tilde{\rho}=\left(\rho_{\mathrm{s}} V_{\mathrm{s}}+\rho_{\mathrm{c}} V_{\mathrm{c}}\right) / V_{\mathrm{T}},
$$

where $V_{s}$ is the volume of the adsorbed layer of surfactant given by $4 \pi\left(R_{\mathrm{T}}^{3}-R_{\mathrm{c}}^{3}\right) / 3$.

It can also be shown that when $\rho_{\mathrm{m}}=\tilde{\rho}$, the contrast between the concentric sphere particle and the dispersion medium is zero and, hence, the intensity of scattering at $Q=O$ reaches either a minimum value reaches either a minimum value or becomes zero. Hence, the value of $\tilde{\rho}$ can be obtained from a plot of $[d \Sigma(O) / d \Omega\rangle]^{1 / 2}$ again $\rho_{\mathrm{m}}$. This is shown in Fig. 3 and gives a value for $\tilde{\rho}$ of $1.45 \times 10^{10} \mathrm{~cm}^{-2}$.

Taking the values of $R_{\mathrm{c}}(40.2 \AA)$ and $\delta(13 \AA)$ together with the values of $\rho_{\mathrm{c}}$ and $\rho_{\mathrm{s}}$ listed in Table 1 gives a value of $\tilde{\rho}$, calculated from Eq. (10) as 1.45 $\times 10^{10} \mathrm{~cm}^{-2}$, which is in good agreement with the experimental value.

From the theoretical discussion of small-angle neutron scattering shown in a previous paper [7], it was shown that a new quantity can be defined to represent the deviation of $\tilde{\rho}$ from the scattering length density of the medium, namely,

$$
\rho=\tilde{\rho}-\rho_{\mathrm{m}},
$$

It then follows that

$$
\begin{aligned}
& R_{g}^{2}=R_{g_{\mathrm{T}}}^{2}+\frac{1}{\rho V_{\mathrm{T}}}\left\{\left(\rho_{\mathrm{c}}-\tilde{\rho}\right) R_{g_{\mathrm{c}}}^{2} V_{\mathrm{c}}\right. \\
& \left.+\left(\rho_{\mathrm{s}}-\tilde{\rho}\right) R_{g_{\mathrm{s}}}^{2} V_{\mathrm{s}}\right\},
\end{aligned}
$$

and for small particles this gives a reasonably linear plot of $R_{g}^{2}$ against $1 / \rho$; (for larger particles see [16]). The results obtained are plotted in Fig. 4. For $1 / \rho=0$ then $R_{g}^{2}=R_{g_{\mathrm{T}}}^{2}$ and, from the figure, this is found to be $1700 \AA^{2}$, giving $R_{g_{\mathrm{T}}}=41.2 \AA^{2}$. Moreover, since $R_{T}^{2}=5 R_{g_{\mathrm{T}}}^{2} / 3$ this gives a value for $R_{T}$ of $53.2 \pm 2 \AA$ which can be compared with the average value obtained from Table 2 . The experimental error in the results made the slope and intercept on the abscissa somewhat more uncertain than the intercept on the ordinate; hence, calculations were only made using the latter.

There appears to be a high degree of self-consistency between the overall particle radius obtained from a compliter fit of the whole curve of

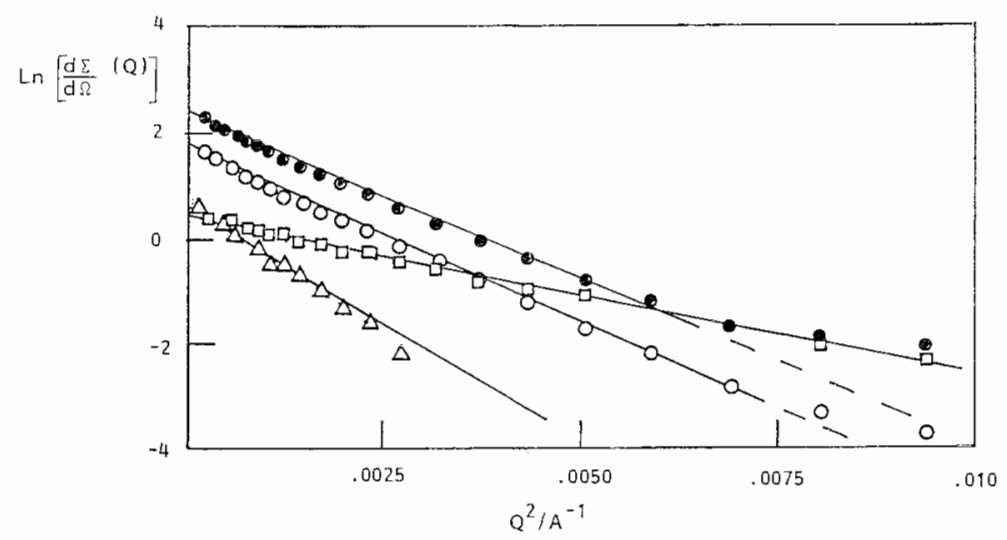

Fig. 2. Curves of $\ln [d \Sigma(Q) / d \Omega]$ against $Q^{2}$ for $1 \% \mathrm{w} / \mathrm{w}$ dispersions of magnesium carbonate in $b_{18}$-octane: $d_{18}$-octane systems. $\square, h_{18}$ -

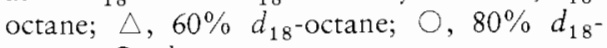
octane;,$d_{18}$-octane. 


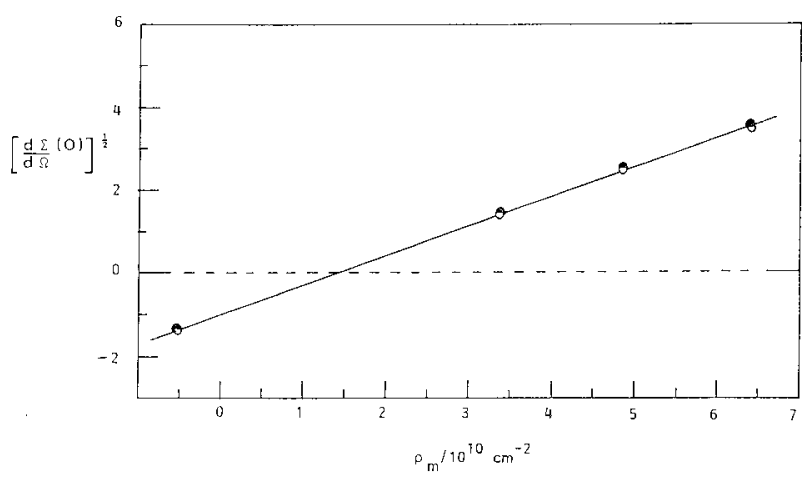

Fig, 3. $[d \Sigma(O) / d \Omega]^{1 / 2}$ against coherent neutron-scattering length of the medium, $\rho_{m} .1 \% \mathrm{w} / \mathrm{w}$ magnesium carbonate dispersions; , from Guinier plots; $\bigcirc$, from fits to complete curve.

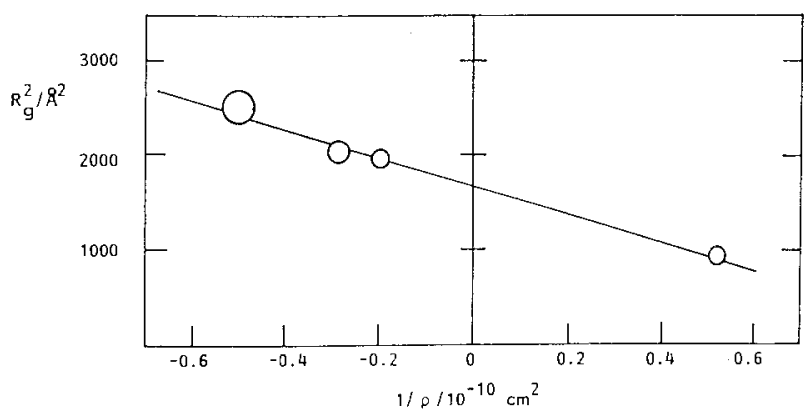

Fig. 4. $R_{g}^{2}$ against $1 / \rho$ for magnesium carbonate dispersions, $1 \% \mathrm{w} / \mathrm{w}$, in hydrocarbon media.

$[d \Sigma(Q) / d \Omega]$ against $Q$ using Eq. (2) and the application of Guinier's law to obtain values of $R_{g}^{2}$, and thence the application of Eq. (12). This suggests that within the approximations imposed by the theory and the error on the experimental data the results provide a useful assessment of the nature of the particle.

\section{Discussion}

In the work described conversion of magnesium oxide into magnesium carbonate by carbonation in the presence of an alkyl aryl sulphonic acid in a nonaqueous medium led to the formation of a stable colloidal dispersion of magnesium carbonate. An interesting feature of the preparative technique is that, whereas calcium carbonate preparations of this type (7) are sensitive to over- carbonation, the conversion of magnesium oxide to the carbonate appears to stop naturally once the amount of carbon dioxide required to form the basic magnesium carbonate has been added. An infra-red examination of the magnesium carbonate dispersion showed a peak in the spectrum at $11.68 \mu \mathrm{m}$ which was assigned to the carbonate. The spectrum was also consistent with the material being amorphous and of composition $3 \mathrm{Mg}$ $\mathrm{CO}_{3} \mathrm{Mg}(\mathrm{OH})_{2} 3 \mathrm{H}_{2} \mathrm{O}(20)$. Thus, this formula was used to calculate the coherent neutron scattering length density of the basic carbonate. As shown in Table 2 this was found to be $3.34 \times 10^{10} \mathrm{~cm}^{-2}$.

Analysis of the small-angle neutron scattering data were made using the core-shell model developed previously $(7,8)$. This model, however, makes a number of assumptions: that the core material is homogeneous, that the shell material is homogeneous, that no changes in the solvency of the shell occur as the dispersion medium changes

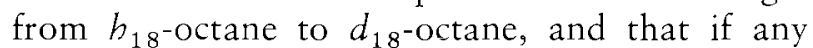
penetration of solvent into the shell occurs it has the same composition as the dispersion medium. It is also assumed that the boundary between the shell and the core is a sharp one. The profile for such a model is illustrated in Fig. 5 for the results obtained, namely, a core radius of $40.2 \pm 1 \AA$ and a shell thickness of $13 \pm 1 \AA$. The values of the coherent scattering length densities taken for the core and the dispersion medium seem to be firmly based. The values for the surface-active agent are more difficult to define precisely. The value taken was

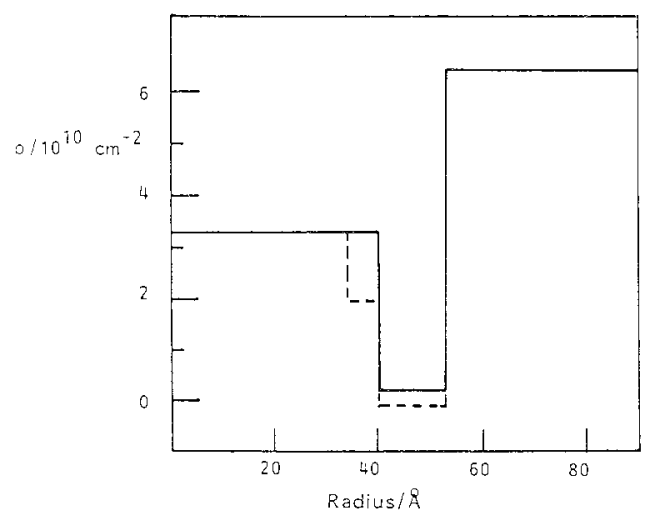

Fig. 5. Coherent scattering length density against distance profile for:- —_, single shell + core model; - _- - double shell + core model. 
that calculated for $\mathrm{C}_{30} \mathrm{H}_{53} \mathrm{SO}_{3}{ }^{-}$, which was 0.25 $\times 10^{10} \mathrm{~cm}^{-2}$. This assumed that the magnesium atoms of the magnesium alkyl-aryl sulphonate were part of the particle.

An alternative model is to assume a core particle and two shells, one of the shells being $\mathrm{Mg}^{2+}$ $\left(\mathrm{SO}_{3}{ }^{-}\right)_{2}$, and the outer shell being the alkyl aryl hydrocarbon $\mathrm{C}_{30} \mathrm{H}_{53}{ }^{-}$. The profile for this model, assuming the coherent scattering length density of $\mathrm{Mg}^{2+}\left(\mathrm{SO}_{3}{ }^{-}\right)_{2}$ to be between $2.26 \times 10^{10} \mathrm{~cm}^{-2}$ (density $=0.90 \mathrm{~g} \mathrm{~cm}^{-3}$ ) and $1.85 \times 10^{10} \mathrm{~cm}^{-2}$ (density $=1.10 \mathrm{~g} \mathrm{~cm}^{-3}$ ) and that of $\mathrm{C}_{30} \mathrm{H}_{53}$ to be $-0.04 \times 10^{10} \mathrm{~cm}^{-2}$ gave the profile shown as a dashed line in Fig. 5; calculations using atomic radii gave $6.8 \AA$ for the length of an $\mathrm{Mg}-\mathrm{O}-\mathrm{S}-\mathrm{O}$ unit. The two-shell model, however, introduces two additional fitting parameters and was not pursued further.

The fits to the core-single shell model gave consistently, over a range of contrasts, a radius of $40.2 \pm 1 \AA$ and a shell thickness of $13 \pm 1 \AA$. This makes it quite clear that the particles have a coreshell morphology and that the overall particle radius is $53 \pm 2 \AA$. Two basically different methods of analysis, that of fitting the experimental results over the whole $Q$-range and that using the Guinier approach gave mutually consistent results. The results were fitted using a polydispersity factor $\sigma_{0}$ of between 0.05 and 0.10 , without significant difference to the fit. This suggested that the particle distribution was rather narrow and is illustrated in Fig. 6, which gives a size-distribution of core radii. The parameters for this distribution were modal core radius $40 \AA$, mean core radius $40.6 \AA$, and

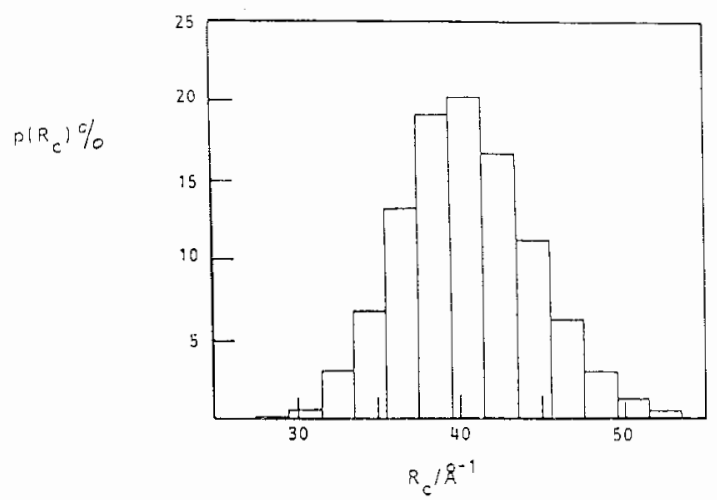

Fig. 6. $p\left(R_{c}\right)$ against $R_{c}$ (see Eq. (7)) for a magnesium carbonate dispersion $(1 \% \mathrm{w} / \mathrm{w})$ in $d_{18}$-octane. $\sigma_{0}=0.10$, giving a standard deviation on the mean of $4.1 \%$.

The shell thickness of $13 \AA$ is smaller than anticipated from earlier work on calcium carbonate dispersions $[7,8]$ where the core particle radius was found to be $22 \pm 2 \AA$ and the layer thickness $18 \pm \AA$. Thus, the core-size found for the magnesium carbonate particles is somewhat larger and the layer thickness somewhat smaller. The reasons for this difference are not completely clear, but it can be speculated that the density of the layer on the magnesium carbonate is lower and this is connected with the presence of the water molecules in the basic carbonate. Since the profile assumed in the model is essentially an "optical" one a lower densiry would affect the thickness obtained, as would the orientation of the molecules at the surface.

From the value of $R_{c}$ for the core $40.2 \AA$, and the layer thickness $13 \AA$, a value of $R_{\text {T }}$ can be obtained and, hence, the total particle volume $V_{\mathrm{T}}$. This was found to be $6.31 \times 10^{-19} \mathrm{~cm}^{3}$, with a core volume of $2.72 \times 10^{-19} \mathrm{~cm}^{3}$ and a shell volume of 3.59 $\times 10^{-19} \mathrm{~cm}^{3}$. Since the molecular mass of the surface-active agent was 500 , it can be calculated that the shell contains 432 molecules. The surface area of the core particle is $2.03 \times 10^{-12} \mathrm{~cm}^{2}$ and, hence, the area occupied per surface-active ion is found to be $47 \AA$. This calculation is inevitably approximate but the value obtained is not unreasonable for a fairly close-packed layer of molecules with the chains exposed to the hydrocarbon medium. In earlier work a computer-simulation of the surfaceactive ion (11) gave an area per molecule of $40 \AA^{2}$ and a minimum length of the chain as ca $16 \AA$; these are close to the values found. The molecule was also found to have a wedge-shaped profile by computer-modelling (17) and, as illustrated earlier $[7,11]$, this may help the molecules to pack on a spherical surface. It is possible that the packing on a magnesium carbonate surface could be slightly different from that of a calcium carbonate surface, but as mentioned above, this is too fine a detail to be drawn from the present results. The suggested tight-packing and the fact that good fits were obtained using the value of $\rho_{\text {s }}$ given in Table 1 would suggest that octane molecules do not extensively penetrate into the shell.

The present work supports the conclusion of our previous work $[2,7-10]$ and the more recent work of others $[18,19]$ that small-angle neutron scatter- 
ing over a wide range of scattering vectors provides a very useful means of characterising dispersions of alkaline earth carbonates of small particle size in nonaqueous media. The brownish colour of the dispersions and the small range of scattering vectors available using time-average light scattering make it difficult to examine these systems by this more conventional technique.

\section{Acknowledgements}

We wish to thank Exxon Chemical Limited and SERC for their support of this work. Our thanks are also due to the ISIS laboratory for the use of neutron facilities.

\section{References}

1. Wood GP (1969) J Inst Petrol 55:194

2. Marsh JF (1987) Chem and Ind 470

3. Booser ER (1981) in: Kirk and Othmer, (eds) Encyclopedia of Chemical Technology, 3rd Edition, Wiley-Interscience $14: 477$

4. Schilling A (1968) Motor Oils and Engine Lubricants, Scientific Publications, G.B. Ltd

5. Koelmans H, Overbeek JThG (1954) Disc Faraday Soc $18: 52$

6. Ottewill RH (1982) in: Goodwin JW, (ed) Colloidal Dispersions, Royal Society of Chemistry, London 197

7. Marković I, Ottewill RH, Cebula DJ, Field I, Marsh JF (1984) Colioid Polym Sci 262:648
8. Marković I, Ottewill RH (1986) Colloid Polym Sci $264: 65$

9. Marković I, Ottewill RH (1986) Colloid Polym Sci 264:454

10. Marković I, Ottewill RH (1987) Colloids and Surfaces 24:69

11. Marković I (1984) Ph.D. thesis, University of Bristol

12. MacDonald IP (1988) Ph.D. thesis, University of Bristol

13. Heenan RK, Osborn R, Stanley HB, King SM, Mildner DFR, Furusaka M (1992) J Appl Cryst (to be submitted)

14. Espenscheid WF, Kerker M, Matijević E (1964) J Phys Chem 68:3093

15. Guinier A (1939) Ann Phys (Paris) 12:161

16. Stuhrmann HB (1974) J Appl Cryst 7:173

17. Davies EK (1982) Chemgraf program suite, Chemical Crystallography Laboratory, University of Oxford

18. O'Sullivan TP, Vickers ME, Heenan RK (1991) J Appl Cryst $24: 732$

19. Vlak WAHM, Mortensen K, Dorrepaal J (1987) Netherlands Energy Research Foundation, Report ECN-197

20. Handbook of Chemistry and Physics (1974), Chemical Rubber Co. Cleveland, p.B-105

Received july 20, 1991; accepted July 25, 1991

Authors' address:

Prof. Dr. R. H. Ottewill

School of Chemistry

University of Bristol

Cantock's Close

Bristol BS8 1TS U.K. 